\title{
A new mutation in the six-domain of SIX3 gene causes holoprosencephaly
}

\author{
L Pasquier ${ }^{1}$, C Dubourg ${ }^{2}$, M Blayau ${ }^{2}$, L Lazaro ${ }^{1}$, B Le Marec ${ }^{1}$, V David ${ }^{2}$ and S Odent ${ }^{1}$ \\ ${ }^{1}$ Services de Génétique Médicale; ${ }^{2}$ Laboratoire de Génétique M oléculaire, CHU Pontchaillou, Rennes, France
}

\begin{abstract}
Holoprosencephaly (HPE) is a severe brain malformation which results from incomplete cleavage of the forebrain during early embryogenesis. The aetiology of HPE is very heterogeneous. Among the genetic factors, SIX3, which is considered to be the functional orthologue of Drosophila genes sine oculis (so) and optix, has been found to be mutated in the homeodomain, in some patients with HPE (HPE2 on chromosome 2p21). We report a new HPE family, presenting a wide spectrum of clinical features, ranging from cyclopia to hypotelorism, in which a mutation was found for the first time in the SIX domain of SIX3: a GG insertion creates a frameshift leading to a nonsense mutation downstream in the homeodomain region. European Journal of Human Genetics (2000) 8, 797-800.
\end{abstract}

Keywords: cyclopia; holoprosencephaly; SIX3; SIX family

\section{Introduction}

Holoprosencephaly (HPE) is a severe brain malformation of the forebrain which results from incomplete cleavage of the forebrain during early embryogenesis. Anatomic brain findings have been defined from the most severe form, alobar HPE with a single ventricle, to lobar HPE in which there is separation of most of the cerebral hemispheres. Phenotype is variable ranging from cyclopia to clinically normal individuals. Ophthalmologic features are prevalent ranging in a continuous spectrum from synophthalmy to a mild hypotelorism. The transmission of isolated HPE is compatible with autosomal dominant inheritance with incomplete penetrance (82\%). ${ }^{1}$

The aetiology is extremely heterogeneous. Among the genetic factors, at least 12 chromosomal loci have been implicated, ${ }^{2}$ including HPE1 in 21q22.3, HPE2 in 2p21, HPE3 in 7q36, and HPE4 in 18p11.3. Many genes have been proposed as candidate genes (see review by Wallis and Muenke ${ }^{3}$ ). But to date, only four of them have been confirmed as HPE-causing genes: Sonic Hedgehog (SHH), ZIC2, SIX 3 and TGIF. SIX $3^{4}$ belongs to the human SIX family which contains several genes exhibiting a strong aminoacid

Correspondence: S Odent, Services de Génétique Clinique et Moléculaire, CHU Pontchaillou, rue Henri Le Guilloux, 35033, Rennes, Cedex 9, France. Tel: +332992842 52; Fax: +33299284174; E-mail: sylvie.odent@chu-rennes.fr

Received 15 March 2000; revised 9 June 2000; accepted 20 June 2000 sequence homology, especially for the SIX and homeodomains, as SIX $1 / S I X 2, S I X 3 / S I X 6$ and $S I X 4 / S I X 5 .^{5}$

$\mathrm{SIX} 3$ has been isolated from the human critical region HPE2 on chromosome2p21, owing to the existence of the mouse Six 3 gene on the homologous distal region of mouse chromosome17. Mouse Six3 is a homeobox gene expressed in the developing retina, lens, hypothalamus, and pituitary; ${ }^{6}$ it has been initially described as sine oculis homologue, a Drosophila gene involved in eye development. A new gene, optix, also expressed in eye primordia of Drosophila embryo, was subsequently identified with a homeodomain 95\% identical to the Six3 protein; ${ }^{7}$ this is the closest paral ogue to SIX 3. Expression of SIX 3 was detected in eye retina in human embryos at 5-7 weeks of gestation until adulthood. ${ }^{5}$

SIX 3 was a good candidate gene due to its expression and its map position overlapping the chromosomal region related to HPE2. Thus Wallis et $\mathrm{al}^{8}$ described four mutations in the homeodomain (one deletion of nine nucleotides and three missense mutations) in patients who had typical HPE facial features.

We have performed a mutational analysis of SIX 3 in $56 \mathrm{HPE}$ patients including those already tested for $\mathrm{SHH}^{9}$ and $\mathrm{ZIC} 2$. We found a mutation located for the first time in the SIX domain in a family with severe ophthalmologic symptoms. In the absence of functional data for previous SIX3 mutations, this novel mutation represents the first lossof-function SIX 3 mutation reported so far. 


\section{Materials and methods}

A total of $56 \mathrm{HPE}$ probands (36clinically sporadic and 20 familial cases) and two cases of unrelated bilateral anophtalmia were studied.

The genomic structure of the human $\mathrm{SIX} 3$ gen $\mathrm{e}^{4}$ was used to design primers pairs covering the entire homeodomain and SIX-domain (Figure1):

Homeodomain: S1 5'-AAG TTC CCG CTG CCA CGC ACC$3^{\prime}$ and S2 5'-GCG GGC CCC GCC ACT AAC-3'

\section{Six-domain: S5 5'-TGT TCC AGC TGC CCA CCC TCA-3' and S6 5'-TAC CAC TCC CGC AAC AGG CTC-3'}

The PCR reactions were performed in a PTC thermal cycler (M) Research, Watertown, MA, USA), with an annealing temperature of $62^{\circ} \mathrm{C}$ for the homeodomain and a temperature gradient from $65^{\circ} \mathrm{C}$ to $56^{\circ} \mathrm{C}$ for the SIX domain.

SSCA was performed with a Genephor system (Pharmacia Biotech, Uppsala, Sweden) in two conditions of migration $\left(20^{\circ} \mathrm{C}\right.$ for $2 \mathrm{~h}$ and $5^{\circ} \mathrm{C}$ for $\left.4 \mathrm{~h}\right)$; the polyacrylamide gels were silver-stained. Heteroduplex PCR products were separated overnight on $50 \mathrm{~cm} \mathrm{MDE}^{\mathrm{TM}}$ (FMC corporation, Rockland, ME, USA), and ethidium bromide stained. ${ }^{10}$

When abnormal SSCA or heteroduplex band shifts were observed, sequencing of the PCR products was performed on the ABI 373A DNA sequencer (Perkin Elmer, Roche, Foster City, CA, USA). Mutations were confirmed by PCR restriction.

\section{Results}

Of the 56 HPE cases for the six and homeodomain of SIX3, anomalies in SSCA and HA were found in one familial case. Direct sequencing showed a GG insertion between nucleo- tides 557 and 558. This mutation creates a frameshift leading to a nonsense mutation (stop codon TAG) 63 aminoacids downstream in the homeodomain region. This mutation was confirmed by PCR restriction with Apal and NlalV.

In this family, three cases with typical features of HPE were described (Figure2). Patient III had synophthalmy and proboscis (Figure3); the autopsy revealed a single optic nerve with coloboma of the retina and confirmed alobar HPE. PatientII2 (Figure4) had cebocephaly with alobar HPE. Foetus II5 (Figure5) was aborted as a result of a singl e cerebral ventricle revealed by ultrasound scan, and cebocephaly which was confirmed by post-mortem examination.

The mutation was identified in a child (II4) presenting with a phenotype typical of the HPE spectrum (single central upper incisor, ocular hypotelorism, mild mental retardation). This DNA alteration was inherited from her father who had only an isolated mild hypotelorism. MRI scans showed no brain malformation in patientII4 or her parents. Unfortunately, no DNA or tissue blocks were available for patients।I1, $1 \mathrm{I} 2$ and $\mathrm{II} 5$.

The mutation was not found in the mother and in the only normal child (II3), nor in 100 normal chromosomes from unrelated Caucasian individuals. In this family, no mutation was identified in $\mathrm{SHH}$ and $\mathrm{ZIC} 2$ genes.

No SSCA or HA anomalies were visualized in the other HPE cases, neither in SIX or homeodomain nor in the two anophtalmic patients.

\section{Discussion}

We have identified a new mutation for the first time in the SIX domain of SIX 3 gene, in one HPE familial case.

The anticipated effect of this mutation could also be associated with HPE for additional reasons: truncation of the

\section{EXON 1}
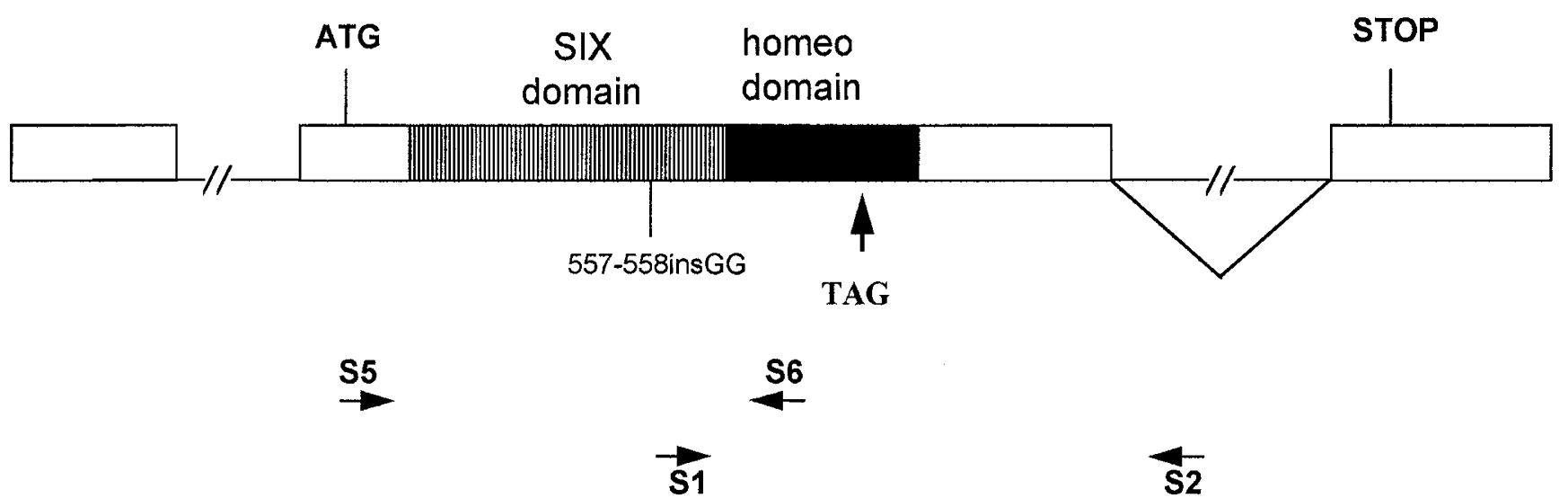

Figure 1 Organisation of the human SIX3 gene (from Wallis et $\mathrm{al}^{8}$ ). Primer sets are indicated by arrows: S5-S6 are used to amplify the SIX domain and S1-S2 for the homeodomain. Bold arrow indicates the position of the stop codon created in the homeodomain by the frameshift mutation 557-558insGG, in the SIX domain. 
A



B

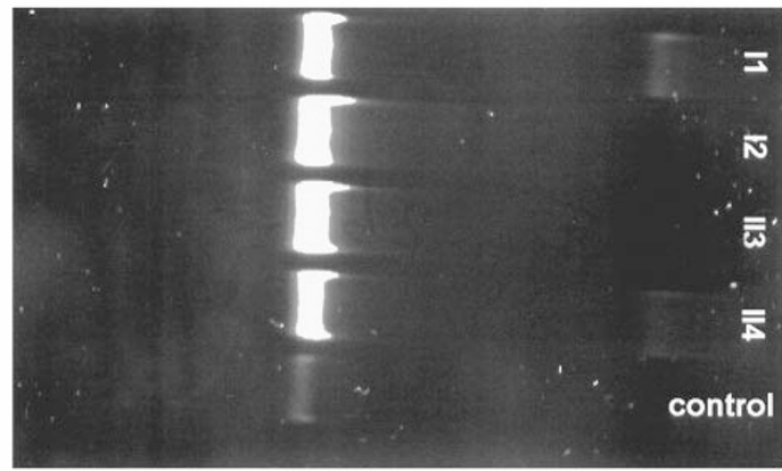

Figure 2 A Pedigree of the HPE family associated with the SIX3 mutation. Filled symbols indicate alobar HPE with cyclopy (II1) or cebocephaly (II2 and II5). Half-filled symbol (II4) indicates minor manifestation with hypotelorism, single upper incisor and mental retardation. Quarter-filled symbol (II) indicates isolated hypotelorism. M: SIX3 mutation carrier; N: no SIX mutation. B Heteroduplex analysis of the SIX domain of SIX in the HPE family.

corresponding protein resulting from the stop codon in the homeodomain; localization in a highly conserved region through evolution of the animal species; ${ }^{8}$ and segregation between the mutation and the associated disease phenotype. An effective functional assay for SIX 3 should be developed to confirm this association, but this novel mutation represents the first truncating mutation and confirms the involvement of SIX3 in HPE.

Interestingly, this $2 \mathrm{G}$ insertion leads to a stop codon downstream in the homeodomain where the four previous mutations (one 9-bp deletion, three missense mutations), had been al ready reported. ${ }^{8}$ The first stop codon described was located in the helix 3 and predicted a change in the DNAbinding helical structure. Wallis et al reported only one missense mutation in helix 3 of the homeodomain, associated with ocular signs (a sporadic case with semi-lobar HPE and associated iris coloboma and micro-ophthal mia). In the family we studied, patients had also ocular features ranging from cyclopia to mild hypotelorism. No specific genotypephenotype association was evident due to the wide variability in these clinical features. Furthermore, such ophthalmologic signs have been described, associated with $\mathrm{SHH}$ mutations. ${ }^{11}$
The variable expression of the $\mathrm{SIX} 3$ mutations might be explained by environmental factors and/or potential modifying genes. This hypothesis was supported by Nanni et al ${ }^{11}$ who reported three patients with both an $\mathrm{SHH}$ mutation and abnormalities in ZIC2 or TGIF genes. In the family we

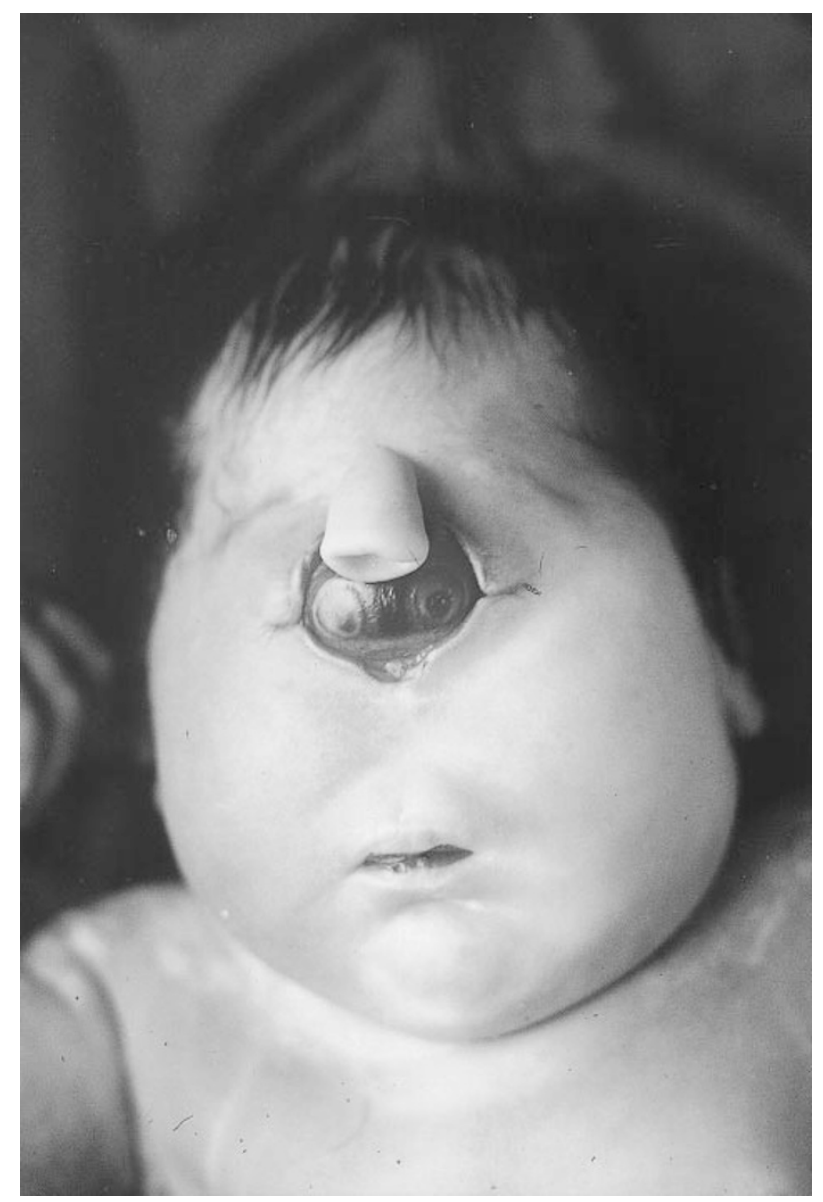

Figure 3 Patient II1: cyclopia with synophtalmy and proboscis.

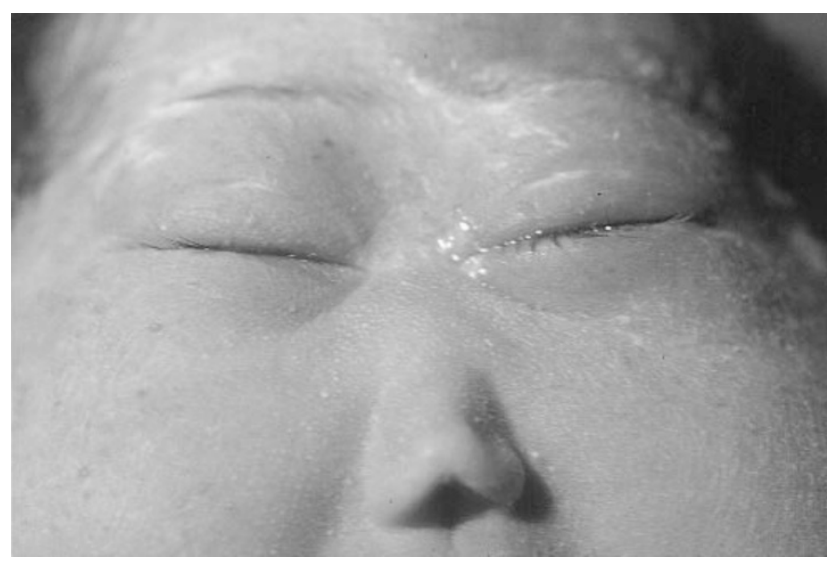

Figure 4 Patient II2: cebocephaly with alobar HPE. 
studied, mutational analysis for $\mathrm{SHH}$ and $\mathrm{ZIC} 2$ genes was negative.

We encountered some recurrent problems during DNA sequencing. The concomitant amplification of another sequence, such as SIX 6, was suggested. This gene, belonging to the SIX family is closely related to $\operatorname{SIX} 3 .{ }^{5}$ In the end the involvement of SIX 6 was excluded by two enzymatic digestions. Furthermore, expression of SIX 6 in the human embryonic eye starts after 12 weeks of gestation. ${ }^{5}$ As human proencephalic brain cleaves into two parts (telencephale and diencephale) at an earlier stage (5 weeks), alterations of SIX 6 should not be involved in HPE. In contrast, SIX 6 haploinsufficency was described in some anophthalmic patients with pituitary anomalies. ${ }^{5}$

The identification of this $\mathrm{SIX} 3$ mutation made genetic counselling more reassuring for the normal male (II3), who

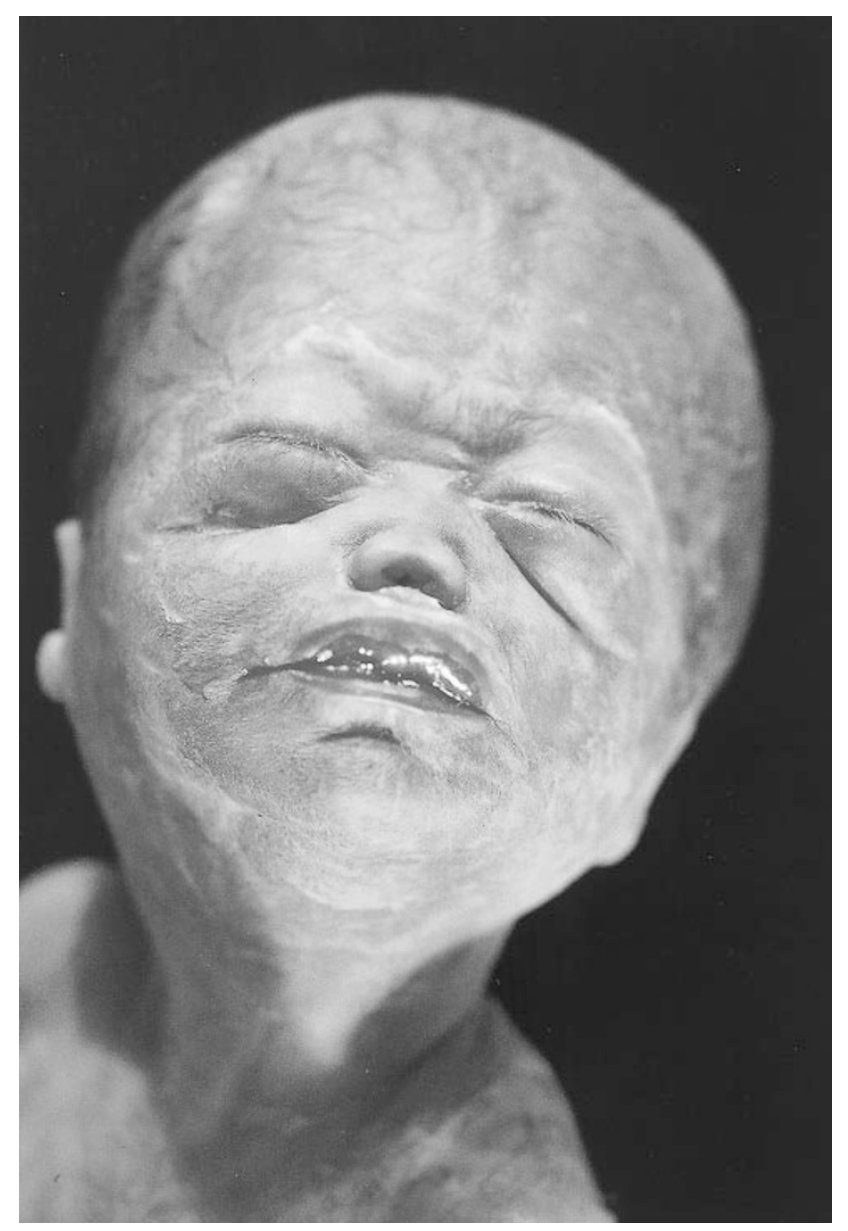

Figure 5 Casell5: medical abortion as a result of single cerebral ventricule. Cebocephaly with alobar HPE. was not a carrier. This SIX 3 mutation, found in a panel of 56 unrelated HPE patients, confirms the well-known genetic heterogeneity of the disease, as five mutations were found in $\mathrm{SHH},{ }^{9,12}$ three in $\mathrm{ZIC} 2$ and a missense mutation in $\mathrm{SIX} 3$ (data not shown). Similar frequencies were described by the previous reports. $^{8,13}$ Finally, this new report is a striking example of the extremely variable expression of the HPE spectrum raising the possibility of modifying genes or environmental factors.

\section{Acknowledgements}

Weare grateful to the families. We thank Brigitte Martinais and Annie Bellamy for technical assistance, and Phillip Jordan for revising the English text. This work was supported by the COREC (CHR Rennes).

\section{References}

1 Odent S, Le Marec B, Munnich A et al: Segregation analysis in nonsyndromic holoprosencephaly. Am J Med Genet 1998; 77: 139-143.

2 Roessler E, Muenke M: Holoprosencephaly: a paradigm for the complex genetics of brain development. J Inherit M etab Dis 1998; 21: 481-497.

3 Wallis DE, Muenke M: Molecular mechanisms of holoprosencephaly. Mol Genet Metab 1999; 68: 126-138.

4 Granadino B, Gallardo ME, Lopez-Rios J et al: Genomic cloning, structure, expression pattern, and chromosomal location of the human SIX3 gene. Genomics 1999; 55: 100-105.

5 Gallardo ME, Lopez-Rios J, Fernaud-Espinosa I et al: Genomic cloning and characterization of the human homeobox gene SIX 6 reveals a cluster of SIX genes in chromosome 14 and associates SIX6 hemizygosity with bilateral anophtalmia and pituitary anomalies. Genomics 1999; 61: 82-91.

6 Oliver G, Mailhos A, Wehr R et al: SIX3, a murine homologue of the sine oculis gene, demarcates the most anterior border of the developing neural plate and is expressed during eye development. Development 1995; 121: 4045-4055.

7 Leppert GS, Yang JM, Sundin OH: Sequence and location of SIX3, a homeobox gene expressed in the human eye. Ophthal Genet 1999; 20: 7-21.

8 Wallis DE, Roessler E, Hehr et al: Mutations in the homeodomain of the human SIX 3 gene cause holoprosencephaly. Nat Genet 1999; 22: 196-198.

9 Odent S, Attié-Bittach T, Blayau M et al: Expression of the Sonic Hedgehog $(\mathrm{SHH})$ gene during early human development and phenotypic expression of new mutations causing holoprosencephaly. Hum Mol Genet 1999; 8/9: 1683-1689.

10 Prior TW, Papp AC, Snyder PJ et al: Heteroduplex analysis of the dystrophin gene: Application to point mutation and carrier detection. Am J Med Genet 1994; 50: 68-73.

11 Nanni L, Ming JE, Bocian $M$ et al: The mutational spectrum of the Sonic Hedgehog gene in holoprosencephaly: SHH mutations cause a significant proportion of autosomal dominant holoprosencephaly. Hum Mol Genet 1999; 8/13: 2479-2488.

12 Odent S, Lazaro L, Blayau M et al: Clinical and molecular study of 56 nonchromosomal unrelated holoprosencephaly cases. European Human Genetics Conference 2000, Amsterdam, 27-30 May 2000.

13 Nanni L, Croen LA, Lammer EJ et al: Holoprosencephaly: Molecular study of a California population. Am J Med Genet 2000; 90: $315-319$. 